\title{
A simple oblique dip model for geomagnetic micropulsations
}

\author{
J. A. Lawrie ${ }^{1}$ and P. N. Smith \\ ${ }^{1}$ School of Mathematical Sciences, University of Sussex, Brighton, E. Sussex BN1 9QH, UK \\ ${ }^{2}$ Department of Physics and Astronomy, University of Sussex, Brighton, E. Sussex BN1 9QH, UK
}

Received: 7 July 1998 / Accepted: 24 August 1998

\begin{abstract}
It is pointed out that simple models adopted so far have tended to neglect the obliquity of the magnetic field lines entering the Earth's surface. A simple alternative model is presented, in which the ambient field lines are straight, but enter wedge shaped boundaries at half a right-angle. The model is illustrated by assuming an axially symmetric, compressional, impulse type disturbance at the outer boundary, all other boundaries being assumed to be perfectly conducting. The numerical method used is checked from the instant the excitation ceases, by an analytical method. The first harmonic along field lines is found to be of noticeable size, but appears to be mainly due to coupling with the fundamental, and with the first harmonic across field lines.
\end{abstract}

Key words. Magnetospheric physics (MHD waves and instabilities).

\section{Introduction}

One of the many complications in a full theory of geomagnetic micropulsations is that the Earth's magnetic field enters the surface obliquely. However, in the box model adopted by Kivelson and Southwood (1986) the field enters everywhere at a right angle because obliquity was considered of minor importance. The same applies even in the hydromagnetic wedge used by Radoski and McClay (1967). An advantage of the right angle assumption is that the field line coordinate can then be removed from calculation apart from a constant, $n$ say, representing the harmonic along field lines that is chosen. The cartesian wedge-shaped model described below has the oblique property, and is

Correspondence to: J. A. Lawrie therefore two dimensional in space, which is rather more awkward; but it was thought time to test the importance of obliquity in a simple case. The model is illustrated in Fig. 1.

Cartesian coordinates $x, y, z$ are chosen, with $x$ representing L-number, $y$ the longitude, and the ambient magnetic field everywhere parallel to the $z$ axis. The boundaries are taken at $x=0, x=D$, and $z= \pm(D / 4+x)$, where $D$ can be taken to be some length typical of the Earth's plasmasphere or magnetosphere. The boundary at $x=D$ is regarded as the outer one, where an excitation can occur, and the others are assumed perfectly conducting. Equations for the MHD axi-symmetric compressional mode can be expressed by the following system of first order derivatives in time $t$ :

$$
\frac{\partial Y}{\partial t}=Q, \quad \frac{\partial Q}{\partial t}=A^{2}\left(\frac{\partial^{2} Y}{\partial x^{2}}+\frac{\partial^{2} Y}{\partial z^{2}}\right) .
$$

Here, $Y$ and $Q$ are the $y$ components of $\boldsymbol{E} / \mu$ and $A^{2} \boldsymbol{j}$ respectively, where $\boldsymbol{E}$ is the electric field, $\boldsymbol{j}$ the electric current, $\mu$ the free space permeability, and $A$ the Alfvén speed assumed constant. In this system, it is easy to see how the $z$ coordinate can be removed from the box model. Recalling that valid $z$ boundaries are then $z=0$ and $z=D$, instead of as above, it can be assumed that both $Y$ and $Q$ have $z$ dependence of form $\sin (\pi n z / D)$. This function is restored after two differentiations and can then be factored out, leaving behind only the constant $-(\pi n / D)^{2}$. The solution cannot then involve any other harmonic (in $z$ ) but the $n$ th, assuming that the excitation has the same $z$ dependence. Another thing shown by Eq. (1) is that the system reduces to the second order wave equation with constant wave speed $A$, and the method given by Sneddon (1957) is valid. This enables the lowest eigenvalues to be checked.

Higher harmonics than those present in the excitation signal are expected to occur. In Fig 1, the incident vertical wave front should enter from the right, and be reflected vertically from the oblique boundaries, so affecting the profile along the field lines. Lee and Lysack (1989) have in fact found higher harmonics along field lines, when using 


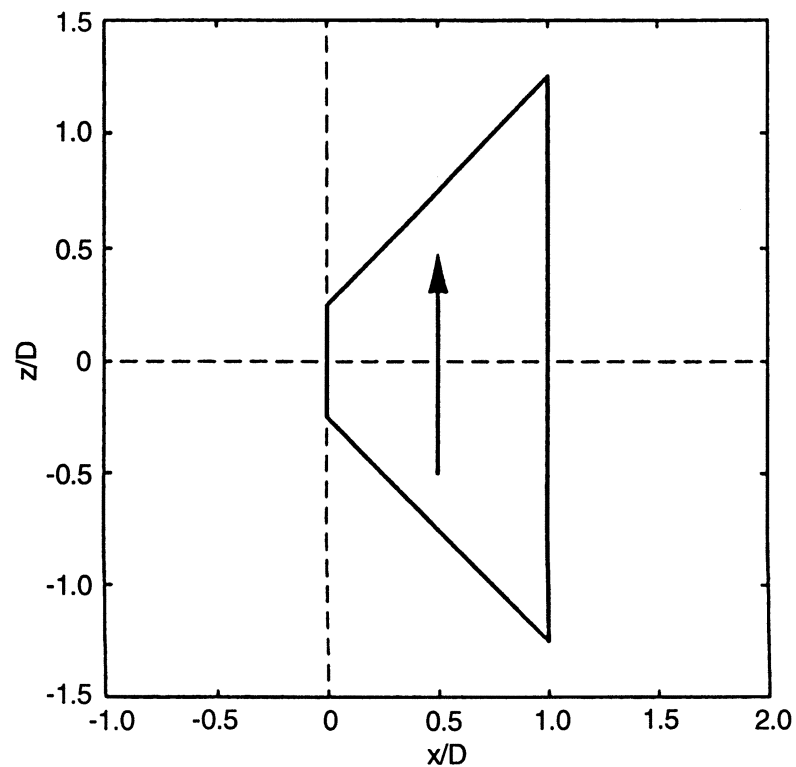

Fig. 1. Cross section of the cartesian wedge model in the normalised $x-z$ plane. The arrow shows the direction of the ambient magnetic field

dipole coordinates; but such harmonics can be produced in various ways, such as something happening at a boundary, or by an appropriate distribution of Alfvén speed. Here, the effect of obliquity only is studied. Itonaga et al. $(1997 \mathrm{a}, \mathrm{b})$ have shown that the $x$ coordinate is very important, in a highly detailed one dimensional study. Accordingly, from the instant excitation is removed, and the boundary condition becomes zero all round the model, the solution is represented by a series of form

$$
Y(x, z, t)=\sum_{k=1}^{\infty} Y_{k}(t) \Phi_{k}(x, z)
$$

where $Y_{k}(t)=\sum_{j=1}^{\infty} C_{j k} \exp \left(i \omega_{j} t\right)$.

$C_{j k}$ is a constant, equivalent to the $C_{i}^{(m)}$ quoted by Sneddon (1957), but not normalised $(m \rightarrow j$ and $i \rightarrow k)$. The first three spatial functions in the former series are:

$$
\begin{aligned}
& \Phi_{1}=\sin (\pi x / D) \cos (\pi z / d), \Phi_{2}=\sin (2 \pi x / D) \cos (\pi z / d) \\
& \quad \text { and } \Phi_{3}=\sin (\pi x / D) \cos (3 \pi z / d),
\end{aligned}
$$

where $d(x)=D / 2+2 x$. It can be seen that $\Phi_{2}$ represents the first harmonic in the $x$ direction across field lines, whereas $\Phi_{3}$ represents the first (even) harmonic along them. In the analytic method, the two series (2) are truncated at $j=k=3$ in order to provide an approximate check of the numerical method. It was assumed that both series converge rapidly. A numerical method is favoured since it is thought to apply in more complicated cases, and only even modes are studied.

\section{Results and discussion}

The Alfvén speed was now assumed to be $D / \pi \mathrm{m} / \mathrm{s}$ everywhere. The excitation was assumed to be at the outer boundary, of form:

$$
\begin{aligned}
Y(D, z, t) & =(1-\cos (2 t)) \cos (2 \pi z / 5 D), \text { for } 0 \leq t<\pi \\
& =0, \text { for } t \geq \pi,
\end{aligned}
$$

all other boundaries being perfect conductors. The excitation thus contained only the fundamental along the outer field line. The conventional four stage RungeKutta method, combined with a finite difference mesh, was used to find a numerical solution to Eq. (1), assuming Eq. (3). The coefficients $Y_{1}, Y_{2}, Y_{3}$, for each value of $t$, were then found by calculating trigonometrical Fourier series with respect to $z$ and then $x$. These were all measured from $t=0$ initially. The solution thus represented was found to be only roughly accurate, using a simple check; but it was thought valid for present purposes. The results found for $Y_{1}$ and $Y_{2}$ are shown in Fig. 2:. These two curves are fairly regular, with angular frequencies approximately $\omega_{1}=$ $1.3 \mathrm{rad} \mathrm{s}^{-1}$ and $\omega_{2}=2.0 \mathrm{rad} \mathrm{s}^{-1}$. The result for $Y_{3}$ is shown in Fig. 3:. This curve is not as regular as those in Fig. 2, and it is therefore necessary to take a (fast) Fourier transform of $Y_{3}$, for $t \geq \pi$ secs, in order to find the frequency content. This is displayed in Fig. 4: The main angular frequencies are, approximately, 1.2, 2.1 and $3.0 \mathrm{rad} \mathrm{s}^{-1}$, and are thought to be $\omega_{1}, \omega_{2}$, and $\omega_{3}$, respectively. The values found by the analytical method are $\omega_{1}=1.3, \omega_{2}=2.2$ and $\omega_{3}=2.7 \mathrm{rad} \mathrm{s}^{-1}$.

In the analytical method, it is clear that the various spatial functions are all coupled with one another when obliquity is introduced. Evidently, the reason that $\omega_{1}$ and $\omega_{2}$ are able to predominate in the Fourier transform of $Y_{3}$ is that $\Phi_{3}$ is coupled to $\Phi_{1}$ and to $\Phi_{2}$. The eigenmodes that mainly correspond to these two spatial functions therefore dominate behaviour, whereas that mainly due to $\Phi_{3}$ itself has little effect. The noticeable

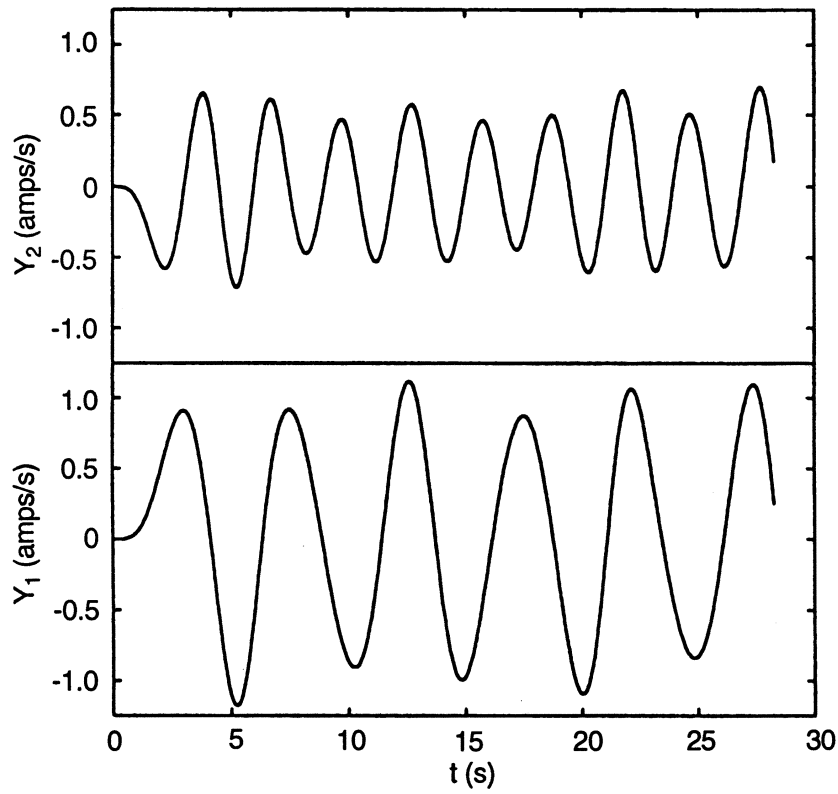

Fig. 2. The calculated values of the harmonics $Y_{1}$ (lower panel) and $Y_{2}$ (upper panel). $Y_{1}$ and $Y_{2}$ have been scaled by a common factor, chosen such that the mean amplitude of $Y_{1}$ is approximately equal to $1 \mathrm{amp} / \mathrm{s}$ 


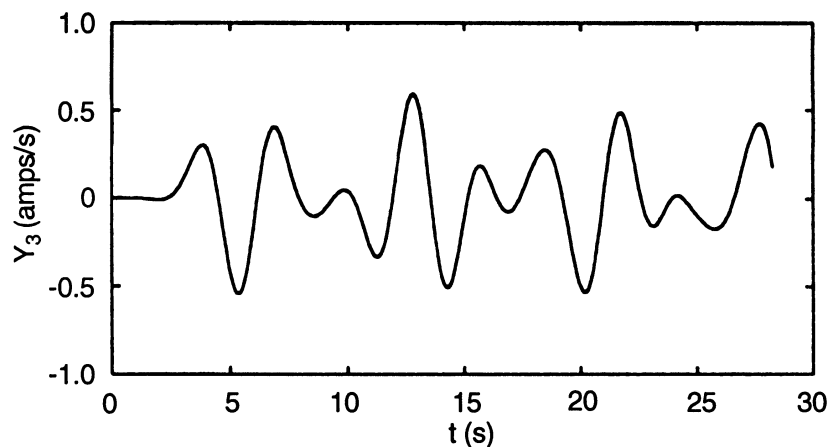

Fig. 3. The calculated value of the harmonic $Y_{3}$. The same scaling factor has been used as for $Y_{1}$ and $Y_{2}$

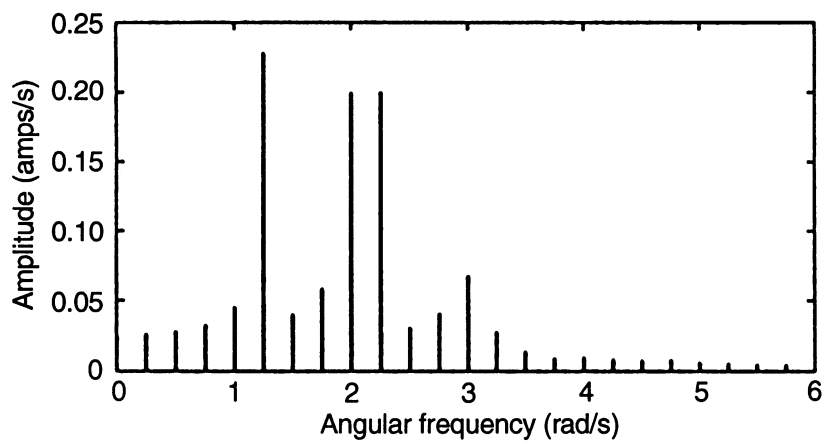

Fig. 4. The Fourier transform of $Y_{3}$ for $t \geq \pi$ s. The Fourier transforms of $Y_{1}$ and $Y_{3}$ have been scaled by a common factor (different from Fig. 2), chosen such that the peak value of the transform of $Y_{1}$ is approximately equal to $1 \mathrm{amp} / \mathrm{s}$

size of $Y_{3}$ must cause first harmonics to appear in the spatial distribution along field lines; but the first harmonic in time would evidently be due rather to spatial variation in the $x$ direction across them. This would be caused by the type of excitation at the outer boundary, which is the major feature.
It would seem also that the numerical method checks reasonably well with the analytical method, from the instant excitation ceases, and the remarks therefore apply equally well to earlier times $(0 \leq t<\pi)$. The method seems useful too, for more complicated cases.

\section{Conclusions}

It is concluded that, in the case considered, the effect of obliquity is to produce a higher spatial harmonic along the field lines. The higher temporal harmonic, on the other hand, seems mainly caused by what happens across the field lines. This seems due to the type of excitation at the outer boundary.

Acknowledgements. The authors are very grateful to Prof. A. Carbery, S. Fujita and to members of the Sussex University Computer Centre, for useful advice.

\section{References}

Itonaga, M., A. Yoshikawa, and K. Yumoto, One-dimensional transient response of the inner magnetosphere at the magnetic equator, 1. Transfer function and poles, J. Geomag. Geoelectr., 49, 21-48, 1997a.

Itonaga, M., A. Yoshikawa, and K. Yumoto, One-dimensional transient response of the inner magnetosphere at the magnetic equator, 2. Analysis of waveforms, J. Geomag. Geoelectr., 49, 49-68, 1997b.

Kivelson, M. G., and D. J. Southwood, Coupling of global magnetospheric MHD eigenmodes to field line resonances, J. Geophys. Res., 91, 4345-4351, 1986.

Lee, D-H., and R. L. Lysack, Magnetospheric ULF wave coupling in the dipole mode: the impulsive excitation, J. Geophys. Res., 94, 17 097-17 103, 1989.

Radoski, H. R., and J. F. McClay, The hydromagnetic toroidal resonance, J. Geophys. Res., 72, 4899-4903, 1967.

Sneddon, I. N., Elements of partial differential equations, McGrawHill, P.230, 1957. 